\title{
Research Facilities
}

National Cancer Institute

\section{Source}

National Cancer Institute. Research Facilities. NCI Thesaurus. Code C18118.

The buildings and equipment used for research. 